\title{
Variations of the Subclavius Muscle: a preliminary study
}

\author{
Wahalawatte $\mathrm{YR}^{1}$, Abeywardhana $\mathrm{UNY}^{1}$ *, Ekanayaka EADA ${ }^{1}$, Dissanayaka $\mathrm{JK}^{1}$ \\ ${ }^{\prime}$ Department of Anatomy, Faculty of Medicine, University of Peradeniya. \\ *niroabey@yahoo.com
}

Variations of the Subclavius muscle have been identified as a cause for Thoracic outlet syndrome (TOS). Knowledge about these variations is particularly important for surgeons as well as radiologists. Twenty eight shoulder pectoral specimens of 14 cadavers were studied in the Department of Anatomy, Faculty of Medicine, University of Peradeniya, Sri Lanka. The skin and the subcutaneous tissues were dissected. The clavicle was lifted up from the medial side. The origins and insertions of the subclavius were documented. Twenty $(71.4 \%)$ male and $8(28.5 \%)$ female specimens were studied. In 2 (7.14\%) specimens, the subclavius muscle was absent.

Twenty six cases showed normal site of origin, that is from the first costochondral junction. Twenty four cases $(85.7 \%)$ had attachments to the clavicle. In 1 case $(3.6 \%)$ an aberrant muscle (subclavius posticus) had gone posterolaterally crossing the subclavian vessels and brachial plexus towards the scapula, without any attachment to the clavicle and inserted in to the transverse scapular ligament, lateral to the inferior belly of omohyoid. In 1 specimen (3.6\%) subclavius coexisted with subclavius posticus where subclavius attached distally to both clavicle and to the coracoid process. This study revealed aberrant attachment of subclavius and aberrant muscle known as subclavius posticus. These can cause TOS due to either compression of the neurovascular structures, narrowing the thoracic outlet and cervico axillary canal. Therefore, this knowledge is important for diagnostic and therapeutic purposes by both surgeons and radiologists. 